\title{
ERRATUM
}

\section{Volume 77, no. 21, 2003, Table of Contents and Author Index and Volume Author Index}

Table of Contents and Author Index: "Marcia V. Fournier" is the correct spelling for the list of authors for the article "Dengue Virus Induces Novel Changes in Gene Expression of Human Umbilical Vein Endothelial Cells,” p. 11822-11832. 